\title{
RENAL TUBERCULOSIS IN PROGRESSIVE STAGE IN A PATIENT PRESENTING WITH RECURRENT DYSURIA, HAEMATURIA AND FEVER: EVALUATION THROUGH CT INTRAVENOUS UROGRAPHY: A CASE REPORT
}

\author{
B. K. Duara ${ }^{1}$,Archita Goel ${ }^{2}$, Rohit Chandak ${ }^{3}$
}

1 Professor and HOD, Department of Radiology, Gauhati Medical College, Guwahati, Assam. 2Junior Resident, Department of Radiology, Gauhati Medical College, Guwahati, Assam. 3Junior Resident, Department of Radiology, Gauhati Medical College, Guwahati, Assam.

\section{ABSTRACT}

A 12-year-old male presented with a 2-month history of recurrent episodes of dysuria, haematuria of 10 days' duration and on and off fever of 4 months duration. There is no history of previous tuberculous infection. Urinalysis revealed leucocyturia and numerous red blood cells, but no growth on multiple urine cultures. The patient showed minimal symptomatic improvement after two courses of nitrofurantoin. Contrast material-enhanced Computed Tomography (CT) of the abdomen was performed for further evaluation of the persistent haematuria and pyuria. Mycobacterium tuberculosis was isolated at acid-fast bacteria urine culture.

\section{KEYWORDS}

CT Intravenous Urography, Renal Tuberculosis, Leucocyturia.

HOW TO CITE THIS ARTICLE: Duara BK, Goel A, Chandak R. Renal tuberculosis in progressive stage in a patient presenting with recurrent dysuria, haematuria and fever: evaluation through CT intravenous urography: a case report. J. Evolution Med. Dent. Sci. 2016;5(49):3173-3176, DOI: 10.14260/jemds/2016/735

\section{INTRODUCTION}

A 12-year-old male patient came to Department of Urology with a chief complaint of a 2-month history of recurrent episodes of dysuria, haematuria of 10 days' duration and on and off fever of 4 months' duration. At physical examination he was pale, febrile and tachypnoeic. Left renal angle tenderness was elicited at palpation. Urinalysis showed an acid urine, leucocyturia, numerous red blood cells and proteinuria. Contrast material-enhanced Computed Tomography (CT) of the abdomen was performed for further evaluation of the persistent haematuria and pyuria. Mycobacterium tuberculosis was isolated at acid-fast bacteria urine culture (Figure 4).

\section{IMAGING FINDINGS}

CT IVU was performed after oral fluid loading and injecting $100 \mathrm{~mL}$ of intravenous contrast material (Iohexol Inj. Omnipaque $350 \mathrm{mgl} / \mathrm{mL}$ ). Pre-contrast plain scan, scans at immediate post injection, 3 mins. and delayed phases were taken. Cross-sectional and MPR images were studied. Unenhanced helical CT of the abdomen demonstrated no obvious calcifications (Figure 1). Contrast-enhanced nephrographic phase CT revealed patchy areas of poorly enhancing renal parenchyma predominantly involving upper and mid pole of left kidney. Cortical thinning was noted in lower and mid poles (Figure 2A, 2C). Asymmetrical dilatation and distortion of the calyceal system was noted (Figure 2B, 2C). Pelvis was not dilated. Pelvis and left ureter were thickened and demonstrated abnormal mural enhancement (Figure 2D). Right kidney was unremarkable. Delayed excretory-phase CT revealed focal narrowing in pelvi-ureteric junction and in the ureter at places (Figure 3A, 3B).

Financial or Other, Competing Interest: None.

Submission 06-01-2016, Peer Review 27-05-2016,

Acceptance 03-06-2016, Published 20-06-2016.

Corresponding Author:

Dr. Archita Goel,

Gauhati Medical College and Hospital,

Bhangagarh, Guwahati, Assam.

E-mail: archita411@gmail.com

DOI: $10.14260 / \mathrm{jemds} / 2016 / 735$
Circumferential wall thickening of the urinary bladder measuring up to $5 \mathrm{~mm}$ was noted. Perivesical fat stranding was noted.

Multiple pockets of collection with enhancing walls were noted in the pelvis. Multiple enlarged lymph nodes were noted in pre- and para-aortic, mesenteric and left renal hilar stations few showing necrosis within.

Renal arteries showed normal course and calibre without significant narrowing. IVC and renal veins were normal in course without any intraluminal thrombus. Prostate was normal for age. Bones revealed no gross abnormality.

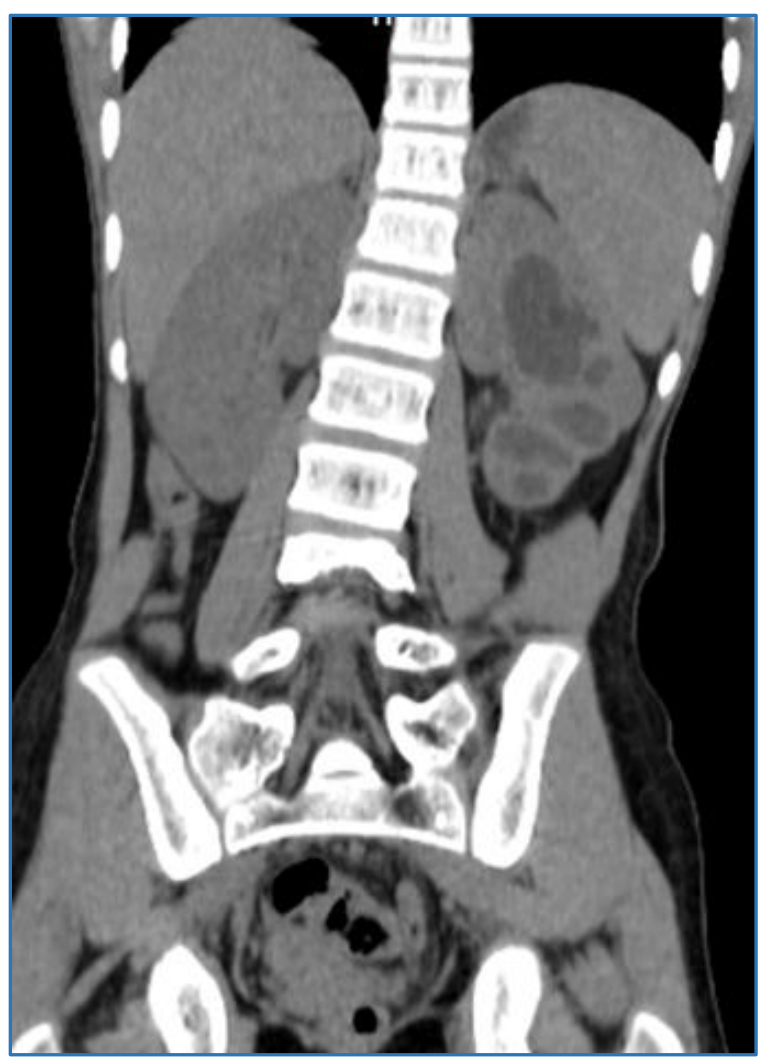

Fig. 1: Pre-Contrast Non-Enhanced CT Reveals No Obvious Calcification 


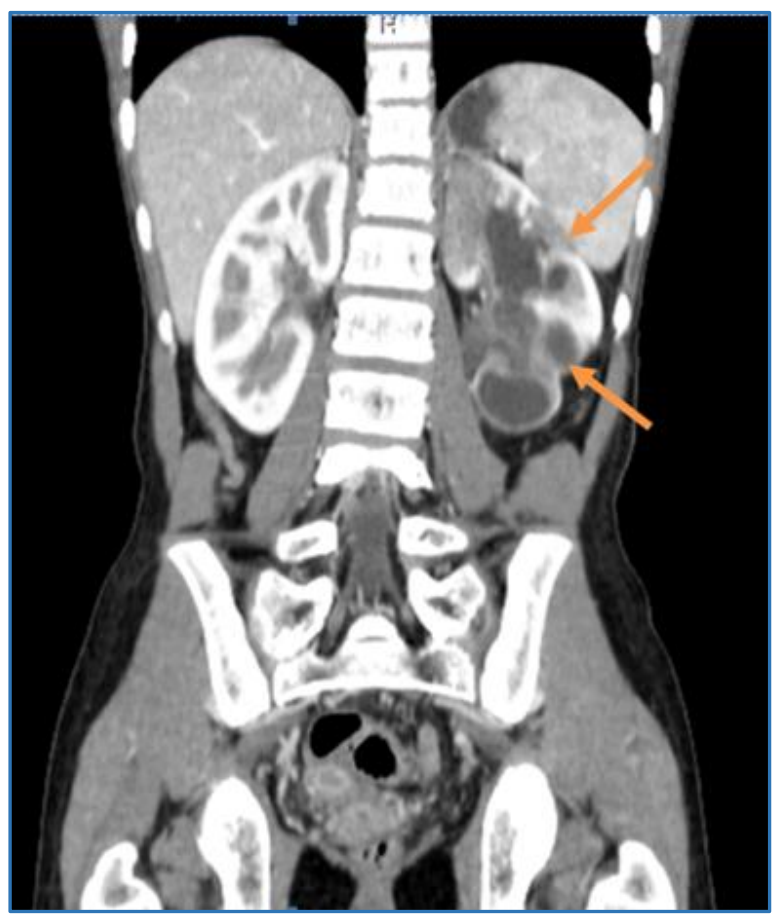

Fig. $2 A$

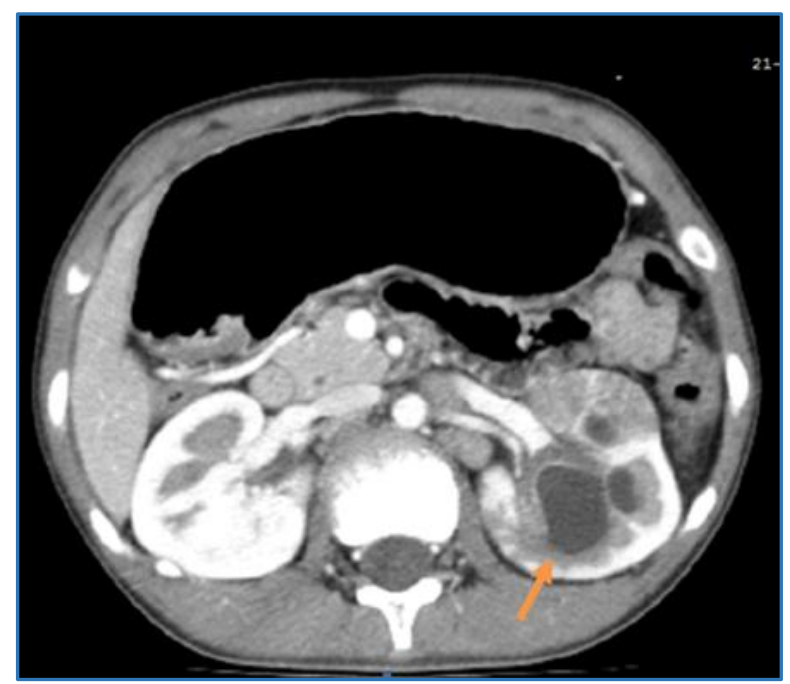

Fig. $2 B$

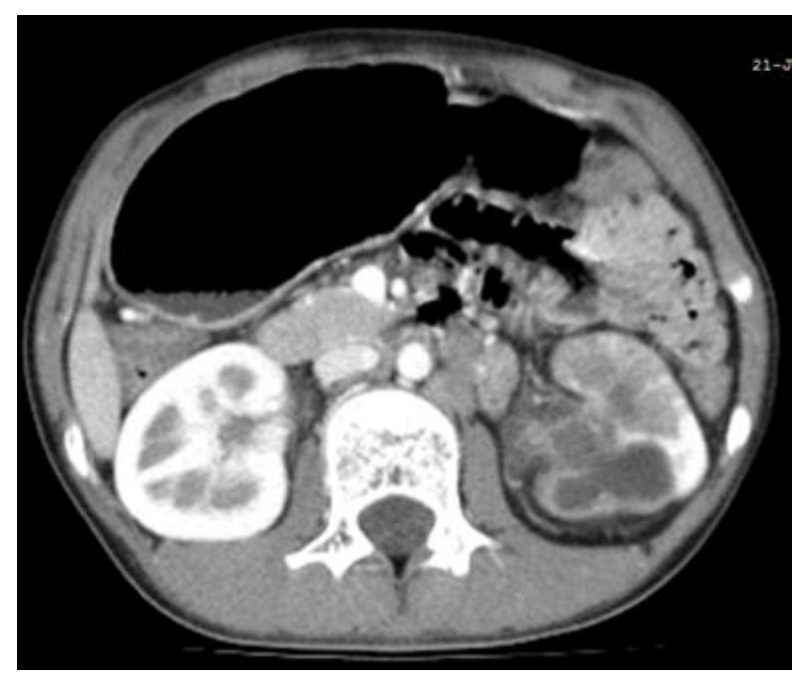

Fig. $2 C$

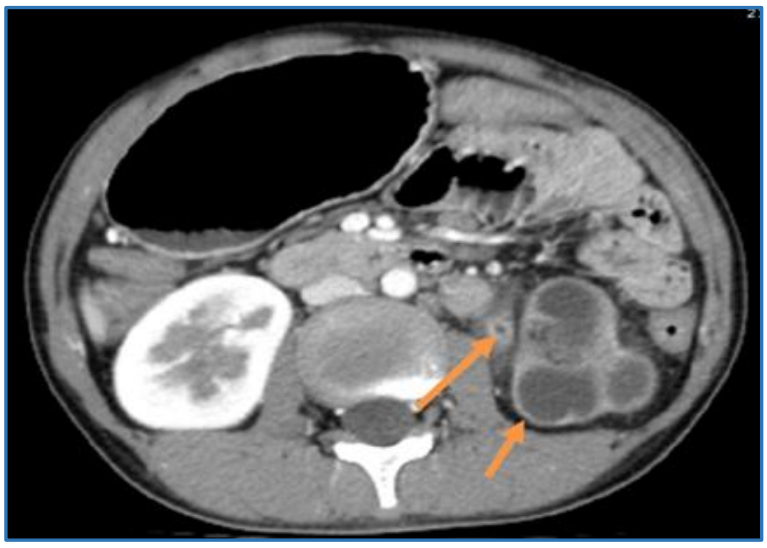

Fig. $2 D$

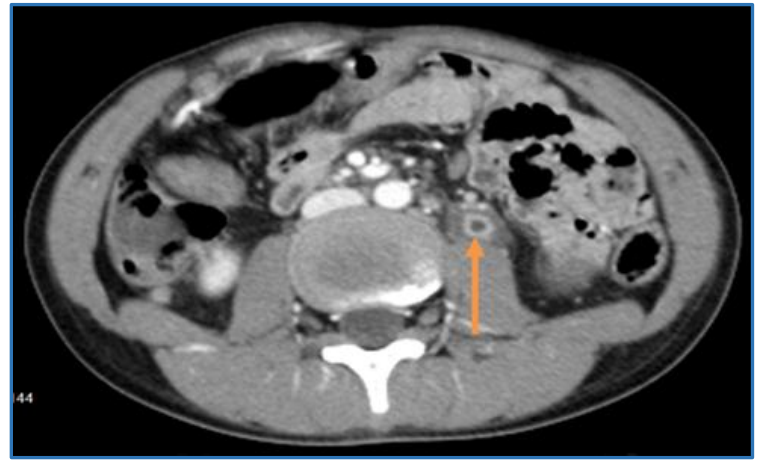

Fig. $2 E$

Figure 2: CT IVU nephrographic phase reveals patchy areas of poorly enhancing left renal parenchyma (A), irregularly dilated and distorted calyces (B, C, D); thickening, focal narrowing at places and abnormal mural enhancement of collecting system including ureter (D, E) and urinary bladder.

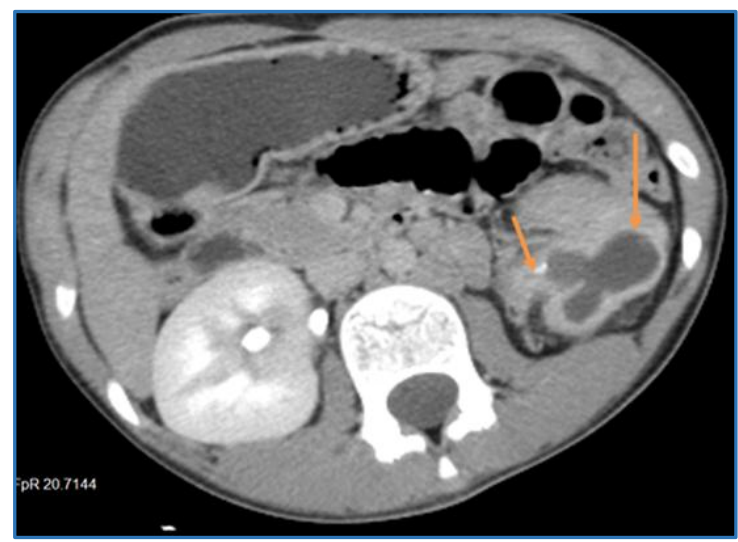

Fig. 3A

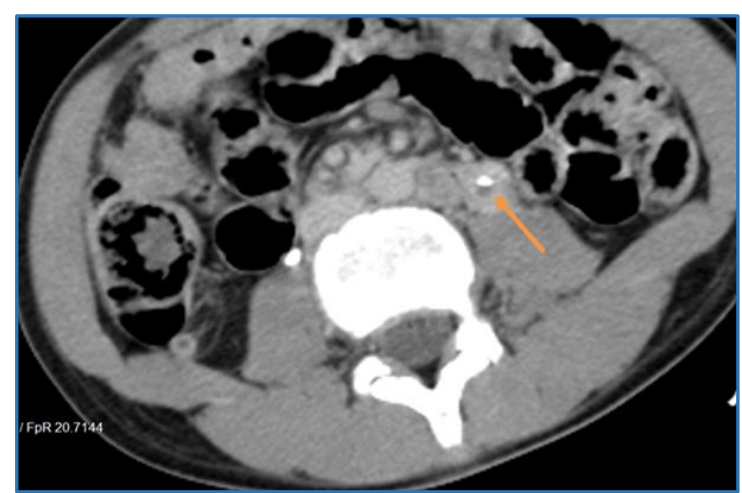

Fig. 3B 
Figure 3: (A) Contrast-enhanced excretory-phase CT scan shows dilated calices and narrowing of pelviureteric junction. (B) Contrast-enhanced excretory-phase CT scan obtained at the level of the mid-ureter shows circumferential left ureteral wall thickening (Arrow). The right ureter is normal.

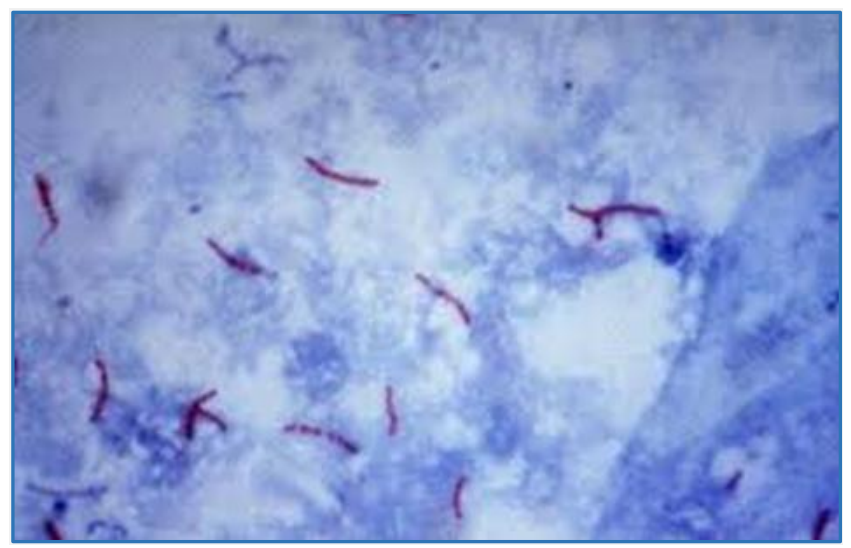

Fig. 4: Photomicrograph showing Mycobacterium Tuberculosis (Ziehl-Neelsen Stain)

\section{DISCUSSION}

Tuberculosis remains the most common worldwide cause of mortality from infectious disease. The World Health Organization estimates that 2 million people die from tuberculosis each year with an additional 6 million people developing active disease. Approximately, one-third of the world's population is believed to harbour latent infection with M tuberculosis. Over $90 \%$ of cases occur in developing countries.

The genitourinary system is one of the most common sites of involvement by extrapulmonary tuberculosis, accounting for $15 \%-20 \%$ of infections outside the lungs. ${ }^{(1,2)}$ Approximately, $4 \%-8 \%$ of patients with pulmonary tuberculosis will develop clinically significant genitourinary infection. $(3,4)$ About $25 \%$ of patients who present with tuberculous genitourinary disease have a known history of prior pulmonary tuberculosis; an additional $25 \%-50 \%$ of patients will have radiographic evidence of prior subclinical pulmonary infection. (4)

Tuberculosis of the kidney results from haematogenous seeding of $\mathrm{M}$ tuberculosis in the glomerular and peritubular capillary bed from a pulmonary site of primary infection. (3-5) Small granulomas form in the renal cortex bilaterally, adjacent to the glomeruli. A high rate of perfusion and favourable oxygen tension increase the likelihood of bacilli proliferating in this location.(4) In patients with intact cellular immunity, there is inhibition of bacterial duplication with confinement of the disease process to the cortex.(6) Multiple bilateral cortical granulomas can remain asymptomatic and dormant for decades. $^{(3-5)}$ In some patients, breakdown of host defense mechanisms leads to reactivation of the cortical granulomas with enlargement and coalescence.(4) Capillary rupture results in delivery of organisms to the proximal tubule and loop of Henle with eventual development of enlarging, caseating granulomas and papillary necrosis.(4) Granuloma formation, caseous necrosis and cavitation are stages of progressive infection, which can eventually destroy the entire kidney. Communication of the granulomas with the collecting system can lead to regional spread of the bacilli into the renal pelvis, ureters, urinary bladder and accessory genital organs. The host's healing response induces fibrosis, calcium deposition and stricture formation, which may contribute significantly to obstruction and progressive renal dysfunction.(4) Despite haematogenous seeding of both kidneys, clinically significant disease is usually limited to one side.(5)

Patients with genitourinary tuberculosis typically have local symptoms including frequent voiding and dysuria. Haematuria can be either microscopic or macroscopic. Symptoms may also include back, flank or abdominal pain.(7-9) Constitutional symptoms such as fever, weight loss, fatigue and anorexia are less common.(7-9) There is often a long latency period (5-40 years) between initial infection and expression of genitourinary disease.(9) Laboratory abnormalities include pyuria, proteinuria and haematuria.(9) Standard urine cultures can be normal. Furthermore, the presence of routine urinary tract pathogens can delay the diagnosis of co-existent tuberculosis. To evaluate for genitourinary tuberculosis, at least three first-morning-void urine samples should be collected for acid-fast staining and mycobacterial cultures. First-morning-void specimens are preferred over 24-hour urine collections because mycobacterial viability decreases with prolonged exposure to acid urine.(4) $M$ tuberculosis is isolated from the urine in $80 \%-$ $95 \%$ of patients with genitourinary tuberculosis. $(8,9)$ Purified protein derivative skin test results will be positive in nearly all patients, but clearly are not specific for genitourinary involvement.

Imaging findings can support the diagnosis of genitourinary tuberculosis, although cultures or histologic analysis is required for definitive diagnosis. Renal calcifications are a common manifestation of tuberculosis at conventional radiography occurring in $24 \%-44 \%$ of patients.(10) Extensive parenchymal calcification in a nonfunctioning, auto-nephrectomised kidney (Putty kidney) is characteristic of end-stage tuberculosis.(11) Calcifications may also be amorphous, granular or curvilinear, typically within the renal parenchyma.(11,12) Focal globular calcification involving an entire renal lobe is frequently associated with a granulomatous mass.(10) Triangular ring-like calcifications within the collecting system are characteristic of papillary necrosis.(13) Other extrapulmonary manifestations of mycobacterial disease, such as mesenteric lymph node and adrenal calcifications as well as spinal abnormalities, may be visible on conventional radiographs. These additional findings can lend support to the diagnosis of renal tuberculosis. ${ }^{(4)}$

Intravenous urography can show a broad range of findings, depending on the severity of infection. Approximately, 10\%$15 \%$ of patients who present with active renal tuberculosis will have normal urographic findings.(14) Parenchymal scars are common, being seen in over $50 \%$ of patients.(10) Irregularity of the papillary tips secondary to necrotizing papillitis ("Moth-eaten" calyces) is an early finding.(10) Small cavities in the papillae can progress to become medullary cavities that communicate with the collecting system.(4) Papillary cavitation results in the spread of infection to the urothelium and submucosa of the draining calyx. A fibrotic reaction develops, which causes stenosis and strictures of the calyceal infundibula.(4) Infundibular strictures can lead to localized caliectasis or incomplete opacification of the calyx (Phantom calyx).(11,15) Some patients may present with generalized hydronephrosis.(15) Scarring can cause sharp angulation of the renal pelvis (Kerr kink).(3) Ureteral 
involvement occurs due to the passage of infected urine. Such involvement first manifests as dilatation and mucosal irregularity (Sawtooth ureter), which may progress with advanced disease to the formation of strictures and ureteral shortening (Pipe-stem ureter).(11) Fusion of multiple strictures may create a long, irregular narrowing. Several non-confluent strictures can produce a "beaded" or "corkscrew" ureter.(3) Reduced bladder capacity is the most common finding in tuberculous cystitis. The bladder may be diminutive and irregular with advanced disease (thimble bladder).(12)

CT is helpful in determining the extent of renal and extrarenal spread of disease.(6,16) CT is the most sensitive modality for identifying renal calcifications, which occur in over $50 \%$ of cases of genitourinary tuberculosis.(3) Coalesced cortical granulomas containing either caseous or calcified material are readily identified at CT. Calices that are dilated and filled with fluid have an attenuation between 0 and $10 \mathrm{HU}$; debris and caseation between 10 and $30 \mathrm{HU}$; putty-like calcification between 50 and $120 \mathrm{HU}$; and calculi greater than 120 HU.(6) Cortical thinning is a common CT finding and may be either focal or global.(6) Parenchymal scarring is readily apparent at CT. Fibrotic strictures of the infundibula, renal pelvis and ureters may be seen at contrast-enhanced CT and are highly suggestive of tuberculosis. CT is not as sensitive as excretory urography in the detection of early urothelial mucosal changes, but is useful in determining the extent of renal and extrarenal spread of infection. $(3,16)$

The current standard of care for drug-sensitive urinary tract tuberculosis in a compliant patient consists of a 6-month regimen of isoniazid and rifampin with pyrazinamide added for the first 2 months.(4) A fourth drug - ethambutol hydrochloride, streptomycin or one of the fluoroquinolones is typically included unless the possibility of drug resistance is exceedingly small.(4) Despite treatment, strictures of the infundibula and ureters can progress secondary to the body's healing response. Surgical intervention is indicated for management of complications including ureteral strictures. (4)

The differential diagnosis for the imaging appearance of renal tuberculosis includes chronic pyelonephritis, papillary necrosis, medullary sponge kidney, calyceal diverticulum, renal cell carcinoma, transitional cell carcinoma and xanthogranulomatous pyelonephritis. $(7,12)$ The most valuable radiologic feature of genitourinary tuberculosis is the multiplicity of abnormal findings. $(3,4,6,16)$

\section{CONCLUSION}

We report a case of renal tuberculosis in progressive stage through CT IVU evaluation in a patient presenting with recurrent dysuria, haematuria and fever, which was initially managed as bacterial cystitis; and hereby conclude that whenever a pattern of chronic renal inflammatory disease is recognized, particularly in the setting of periureteric or peripelvic fibrosis, tuberculosis must be considered clinically.

\section{REFERENCES}

1. World health organization. Stop TB annual report 2001 Geneva, Switzerland: World Health Organization, 2002.

2. Farer LS, Lowell AM, Meador MP. Extrapulmonary tuberculosis in the United States. Am J Epidemiol 1979;109(2):205-17.

3. Leder RA, Low VH. Tuberculosis of the abdomen. Radiol Clin North Am 1995;33(4):691-705.

4. Pasternak MS, Rubin RH. Urinary tract tuberculosis. In: Schrier RW (eds). Diseases of the kidney and urinary tract, $7^{\text {th }}$ ed. Philadelphia, Pa: Lippincott Williams \& Wilkins, 2001:1017-37.

5. Medlar EM. Cases of renal infection in pulmonary tuberculosis: evidence of healed tuberculosis. Am J Pathol 1926;2(5):401-13.

6. Goldman SM, Fishman EK, Hartman DS, et al. Computed tomography of renal tuberculosis and its pathological correlates. J Comput Assist Tomogr 1985;9(4):771-6.

7. Narayana A. Overview of renal tuberculosis. Urology 1982;19(3):231-7.

8. Simon HB, Weinstein AJ, Pasternak MS, et al. Genitourinary tuberculosis: clinical features in a general hospital population. Am J Med 1977;63(3):410-20.

9. Christensen WI. Genitourinary tuberculosis: review of 102 cases. Medicine (Baltimore) 1974;53(5):377-90.

10. Kollins SA, Hartman GW, Carr DT, et al. Roentgenographic findings in urinary tract tuberculosis: a 10 year review. Am J Roentgenol Radium Ther Nucl Med 1974;121(3):487-99.

11. Engin G, Acunas B, Acunas G, et al. Imaging of extrapulmonary tuberculosis. Radio Graphics 2000;20(2):471-88.

12. Harisinghani MG, McLoud TC, Shepard JA, et al. Tuberculosis from head to toe. Radiographics 2000;20(2):449-70.

13. Davidson AJ, Hartman DS, Choyke PL, et al. Parenchymal disease with normal size and contour. In: Davidson AJ (eds). Davidson's radiology of the kidney and genitourinary tract $3^{\text {rd }}$ ed. Philadelphia, Pa: Saunders, 1999:327-58.

14. Kenney PJ. Imaging of chronic renal infections. AJR Am J Roentgenol 1990;155(3):485-94.

15. Roylance J, Penry JB, Davies ER, et al. The radiology of tuberculosis of the urinary tract. Clin Radiol 1970;21(2):163-70.

16. Wang LJ, Wong YC, Chen CJ, et al. CT features of genitourinary tuberculosis. J Comput Assist Tomogr 1997;21(2):254-8. 